\title{
Low Energy Dart Test for Mechanical Evaluation of Ophthalmic Materials
}

\author{
Antonio B. Martínez*, Pau Artús ${ }^{\dagger}$, Juan C. Dürsteler*, and David Arencón*
}

\begin{abstract}
Purpose. Many impact tests fail to rigorously analyze the polymer behavior at impact, because they are performed in an energy range too different from real-life incidents, use specimens with other geometries than those of their final application, or they do not take in account polymer viscoelastic nature. A novel low energy impact method that overcomes current method limitations is presented for ophthalmic polymers and advances our understanding of the behavior of these materials under impact conditions.

Method. Plate-shaped specimens of two known materials, CR-39 and Superfin, were tested in an energy range around their failure limit. A non-conservative model was proposed to predict the dynamic response of the specimens that did not fail. Both the deflection and indentation mechanisms were introduced in the model, which was solved using a fourth order Runge-Kutta numerical method. Damper coefficients that were introduced to model the energy dissipation and elastic modulus were obtained after the fitting process. Rupture stress and absorbed energy at failure were obtained from the specimens that failed.

Results. Very good agreement between experimental and calculated data was observed. Under non-failure conditions, Superfin and CR-39 showed similar elastic modulus, although slightly larger energy dissipation was observed for CR-39. However, Superfin clearly outperformed CR-39 when measuring rupture stress and absorbed energy at failure with values $54 \%$ and $170 \%$ larger, respectively.

Conclusions. Low energy impact methods are a very powerful tool to study and compare ophthalmic materials. The model satisfactorily predicted the behavior of materials in low energy impact conditions and can be used to obtain critical material characteristics. In this particular case, the method was used to quantify mechanical differences among CR-39 and Superfin. Of these two, the latter is the best performing material.
\end{abstract}

(Optom Vis Sci 2009;86:979-987)

Key Words: polymer, ophthalmic, low energy impact, non-conservative model, CR-39, Superfin

M any industrial fields are taking advantage of the latest developments of the polymers that are regularly being introduced in the market. Ophthalmic lenses have arisen as one of these very specialized sectors with its own selection of thermosetting and thermoplastic polymers designed to optimize both optical and mechanical properties required for this application.

Although CR-39 resin has been the most successful material in the ophthalmic lens production since Armorlite Company introduced it in 1947, a wide variety of new materials have been appearing during the past 20 years that have higher refractive index and, in some cases, are claimed to show better mechanical properties.

Mechanical tests used to compare polymer materials do not always take in account the final application and are often per-

\section{$* \mathrm{PhD}$}

${ }^{\dagger} \mathrm{MSc}$

Indo Lens Group S.L., Sant Cugat del Vallès, Spain (PA, JCD), and Centre Català del Plàstic, Terrassa, Spain (ABM, DA). formed under conditions that differ substantially from the geometry and final use of the product. ${ }^{1}$ For many materials, this may not be of special relevance but in the case of polymers, the mechanical properties depend not only on the temperature but also on the strain rate because of their viscoelastic nature. ${ }^{2}$ Indeed, as the strain rate increases, yield stress also increases, strain decreases, and the energy that the material can absorb drastically drops.

Classical methods that measure the absorbed energy during impact, such as such as Izod or Charpy pendulums, are uniaxial tests that use notched specimens. ${ }^{3,4}$ These tests are performed at moderate impact velocities and require specimen geometries that greatly differ from ophthalmic lenses, which can be critical because results strongly depend on the specimen geometry and test setup. In these tests, the available energy on the impact head always exceeds the amount the specimen can absorb.

The American Food and Drug Administration defined a very basic method to analyze impact resistance of lenses. ${ }^{5}$ What is com- 
monly known as the "drop-ball test" consists of a steel ball falling from a fixed height onto a lens placed on a rubber ring support. This test was originally designed as a production tool for mineral lenses to ensure correct and safe thermal tempering process, and it is actually supposed to be statistically applied to each commercialized lot of lenses. Although the drop-ball configuration is multiaxial and therefore more realistic than pendulum tests, it gives little information about material characteristics besides a statistical pass-fail verdict. In the field of safety eye wear, similar tests have been developed to analyze impact resistance of non-coated and coated materials, mainly polycarbonate, to blunt- and sharp-headed striker at extremely high speeds. ${ }^{6}$ However, few properties of the material can be quantified from these experiments besides another passfail qualification at a particular striker velocity and energy.

Other more elaborated falling weight tests can calculate the impact energy at $50 \%$ chance of sample failure. Because the result corresponds to the average energy needed to break one-half of the specimens, this represents an improvement compared with other tests, but unfortunately, considerable numbers of specimens are needed and no information about the material mechanical behavior is obtained.

The evolution of electronics lasting recent years has allowed improvement of data acquisition by placing a piezoelectric sensor inside the falling dart head, very close to the impact point. Then, the signal is amplified and stored at a frequency of $1 \mathrm{MHz}$ in a computer. This instrumentation of the impact testing has proven to be very useful to understanding the impact behavior. The whole impact is being captured and the load vs. time data can be analyzed. The impact energy can be modified by controlling the drop mass or the falling height. For instance, the given energy can be far beyond the breakage limit or, alternatively, the equipment can work in a smaller energy range around the specimen failure limit.

In the common practice for impact tests, the impact energy is greater than necessary energy for fully breaking of the specimen. However, the lower energy impacts ${ }^{7}$ are a subject of importance in the case of polymers and composites because of the resulting invisible damage caused by even mild impacts. This damage caused in the specimen could be modified by introducing a slight variation in the striker energy. As a result, the material characterization is easily performed.

The setup involves a falling hemispherical tipped dart colliding against plate-shaped specimens simply supported on a metallic ring. Becasue mechanical performance of polymers strongly depends on the specimen geometry, a shape closer to a lens geometry results in an improvement over standard pendulums. In addition, the specimen can be directly casted to the appropriate geometry and does not need to be mechanized for the experiment. This avoids the generation of small scratches and cracks that could alter the test results. Furthermore, the technique could potentially be used to test real lenses, and although not providing the same amount of information, it would be possible to at least classify the lens material behavior according to its typology (Fig. 1). Materials that have similar toughness, i.e., they absorb the same amount of energy, which can reach the failure limit at longer or shorter deformations and loads.

The specimen behavior during impact could be separated in two parts: the contact with the falling dart and the plate deflection. The contact with the dart head results in an instantaneous pressure of

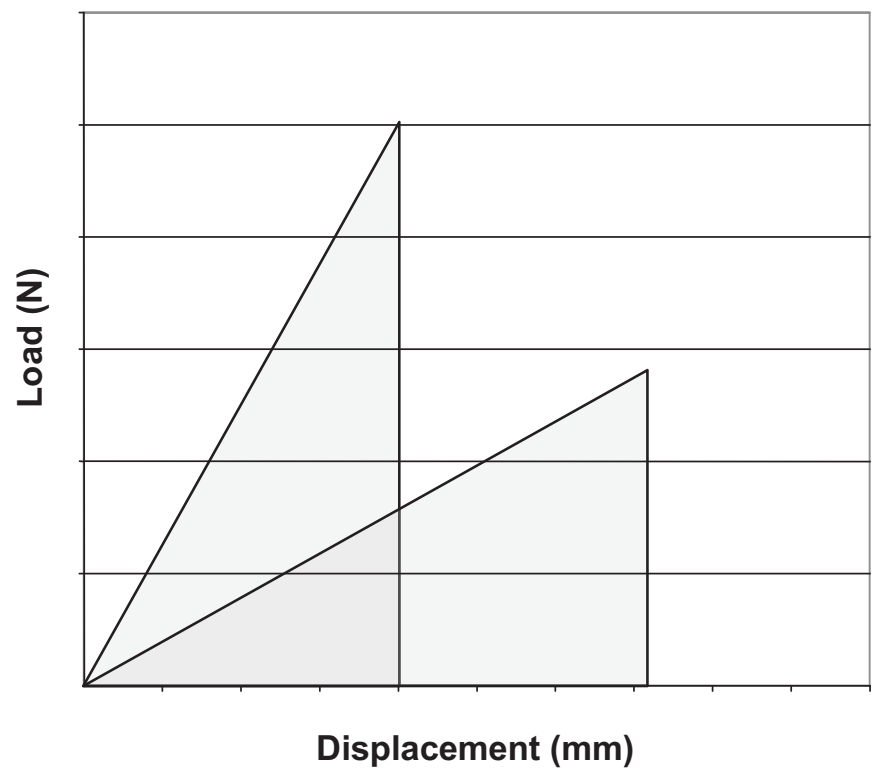

FIGURE 1.

Load vs. displacement for two types of material behavior showing equal amount of absorbed energy (gray area) but different elongation and maximum load.

the compressed area, which in turn creates a local deformation over the plate surface in the form of an indentation. As shown in previous work, ${ }^{8}$ the contribution of this part can be studied separately using a specific experimental setup and its corresponding mechanical model based on the Hertz law. ${ }^{9}$ The specimen deflection can be modeled with the theory of small deflection of plates. ${ }^{10}$

Although many published models for predicting the behavior of materials during low energy impacts in flexed plate configuration are conservative, ${ }^{11-13}$ i.e., they assume no energy loss, the dynamic response of materials in such conditions can never be considered fully elastic. Consequently, a better approach is founded by the non-conservative methods that are based on lumped mass-spring models. $^{14,15}$

A more simplified procedure for predicting indentation, force history, and impact duration throughout the impact on plates is developed in this article based on a lumped mass spring model. The differential equations governing the striker-plate system can be solved by fourth order Runge-Kutta numerical methods. Subsequently, the impact response can be calculated.

As an example, the method was tested on two apparently similar polymers used in the ophthalmic field, i.e., CR-39 and Superfin ${ }^{\circledR}$, and revealed differences in their behavior under these test conditions showing that this technique and model can be a very useful tool for research into material mechanical properties. The method has already been successfully applied to other polymers and composite materials. ${ }^{16-18}$

\section{EXPERIMENTAL}

\section{Method}

Impact tests were performed using a striker mounted in a Ceast Dartvis apparatus with a $12.7 \mathrm{~mm}$ diameter hemispherical dart head. Two drop masses of 0.503 and $1.504 \mathrm{~kg}$ were used. The sample plates were simply supported on a metallic ring of $30-\mathrm{mm}$ 


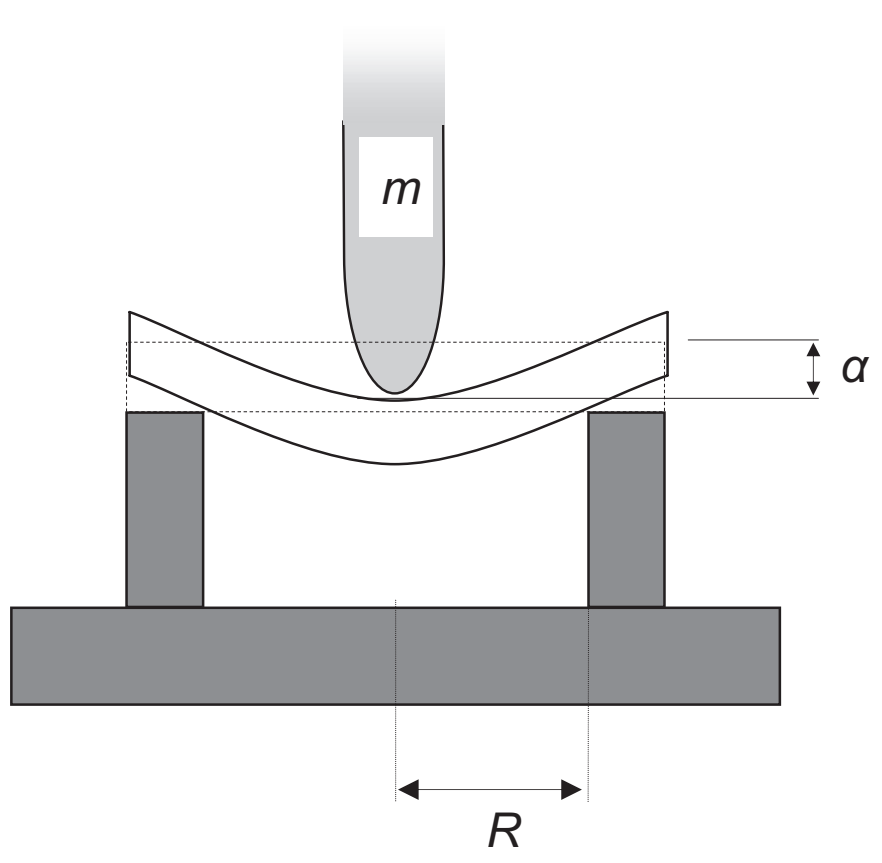

FIGURE 2.

Scheme of the experimental setup.

internal radius $\mathrm{R}$, as shown in Fig. 2. Load vs. time data was acquired for $5 \mathrm{~ms}$ at a scanning frequency of $1 \mathrm{MHz}$ (1 million values per second). Impact velocity $\left(\mathrm{v}_{0}\right)$ was measured using a velocity photodetector.

Registered load $\mathrm{F}$ as a function of time $\mathrm{t}$ was interpreted using Newton's second law (Eq. 1), where $\ddot{\alpha}$ is the acceleration of the $\mathrm{dart}, \mathrm{m}$ its mass, and $\mathrm{g}$ the gravity acceleration.

$$
\mathrm{F}(\mathrm{t})=\mathrm{m} \ddot{\alpha}+\mathrm{mg}
$$

By integrating two times Eq. 1, the displacement $\alpha$ of the dart can be calculated (Eq. 2).

$$
\alpha=v_{0} \mathrm{t}-\frac{1}{\mathrm{~m}} \int_{0}^{\mathrm{t}} \int_{0}^{\mathrm{t}} \mathrm{F}(\mathrm{t}) \mathrm{dt}^{2}+\frac{1}{2} \mathrm{gt}^{2}
$$

Experimentally, $\alpha$ was calculated by numerical integration of the load vs. time curve.

\section{Restitution Coefficient}

The restitution coefficient $\epsilon$ is an empirical parameter that measures the energy loss during the impact. As shown in Eq. 3, it is defined as the quotient between the velocity immediately after $\mathrm{v}_{1}$ and before $\mathrm{v}_{0}$ the dart contact.

$$
\varepsilon=\frac{v_{1}}{v_{0}}
$$

Because the kinetic energy dissipated during a dart impact can be calculated by subtracting the final energy $E_{1}$ from the initial energy of the dart $\mathrm{E}_{0}$, this energy loss can be written as:

$$
\mathrm{E}_{\mathrm{diss}}=\mathrm{E}_{0}-\mathrm{E}_{1}=\frac{1}{2} \mathrm{~m} v_{0}^{2}-\frac{1}{2} \mathrm{~m} v_{1}^{2}
$$

When Eqs. 3 and 4 are combined, the dissipated energy can be written in the form:

$$
\mathrm{E}_{\mathrm{diss}}=\frac{1}{2} \mathrm{~m} v_{0}^{2}\left(1-\varepsilon^{2}\right)
$$

In material science, the restitution coefficient is associated with elastic and inelastic behavior of the studied material. As can be deduced from Eq. 5, when this coefficient equals 1, there would be no energy dissipation during impact and the material response would be considered completely elastic. On the other hand, when the restitution coefficient equals 0 , the material response is totally inelastic and the energy dissipated would equal the initial kinetic energy of the dart $\mathrm{E}_{0}$. However, real cases always fall in between.

Restitution coefficient can also be calculated by equating impulse and momentum as follows:

$$
\int_{v_{0}}^{v_{1}} \mathrm{~m} v=\int_{0}^{\mathrm{t}} \mathrm{Fdt}
$$

Integrating Eq. 6 and rearranging with Eq. 3 taking in account $\mathrm{v}_{0}$ and $\mathrm{v}_{1}$ have opposite signs, this parameter can be written as:

$$
\varepsilon=\frac{\int_{0}^{\mathrm{t}} \mathrm{F}(\mathrm{t}) \mathrm{dt}}{\mathrm{m} \nu_{0}}-1
$$

Therefore, the restitution coefficient can experimentally be calculated with Eq. 7 numerically integrating the load vs. time curve.

\section{Specimens}

Two sets of CR-39 and Superfin sample plates were synthesized using standard thermal polymerization.

a. CR-39 plates of $70 \mathrm{~mm}$ diameter and $4.6 \mathrm{~mm}$ nominal thickness were polymerized from diethyleneglycol bisallylcarbonate (ADC) by Acomon used as received after the addition of 3\% wt. of an initiator, such as isopropyl peroxycarbonate (IPP) also by Acomon. The monomer was stirred for $30 \mathrm{~min}$, filtered, and injected into a cavity formed by two flat glass molds joined through adhesive tape. A thermal cycle from 40 to $90^{\circ} \mathrm{C}$ in $22 \mathrm{~h}$ was applied. After demolding, specimens were annealed for $1 \mathrm{~h}$ at $94^{\circ} \mathrm{C}$.

b. Superfin is a proprietary polymer of Indo composed by $50 \%$ of ADC and $50 \%$ of an aromatic polyester oligomer of high molecular weight terminated by reactive allyl groups. Superfin plates of $70 \mathrm{~mm}$ diameter and $4.6 \mathrm{~mm}$ nominal thickness fabricated through an analogous polymerization process to CR-39 were supplied by Indo Lens Group S.L.

\section{MODEL}

The transversal collision between a rigid striker and a specimen made of a uniform material with linear elastic behavior can be modeled by a mass-spring system. If the plate mass is very small in front of the drop mass, then the spring can be considered mass less and the system is reduced to a single degree of freedom. 
When the plate is relatively thin (the thickness/diameter ratio is smaller than 0.2), the thin plate theory can be applied and the contribution of shear deformation can be neglected. When the deflection of the plate is relatively small (the deflection/thickness ratio is smaller than 0.5 ), the small deflection theory can be applied and the influence of the membrane stretching can be neglected. In this article, the thin plate small deflection theory was used because the tested specimens and the resulting deflections satisfied these criteria.

For analysis purposes, the impact event is usually split into two parts, a localized contact and an overall plate deflection. Surface indentation is assumed to follow Hertzian contact law and it is composed by a non-linear spring. ${ }^{9}$ Complete models may be used to exactly describe the deformation of the target using plate theory for simple cases, such as small deflections. ${ }^{10}$

Because almost all real systems are not conservative to a greater or lesser degree, a better approach can be expected by means of non-conservative systems. With this aim, two dashpots can be introduced into the conservative system, one in a serial disposition for the energy dissipation in the contact and another one in a parallel disposition for the energy dissipation during the plate deflection.

The model is shown in Fig. 3. The set of differential Eqs. 1 and 8 to 13 describe the system motion.

$$
\begin{gathered}
\alpha=\alpha_{1}+\alpha_{2}+\alpha_{3} \\
\mathrm{~F}_{\mathrm{K}_{\mathrm{i}}}=\mathrm{K}_{\mathrm{i}} \alpha_{1}^{3 / 2} \\
\mathrm{~F}_{\mathrm{C}_{\mathrm{i}}}=\mathrm{C}_{\mathrm{i}} \dot{\alpha}_{2}
\end{gathered}
$$

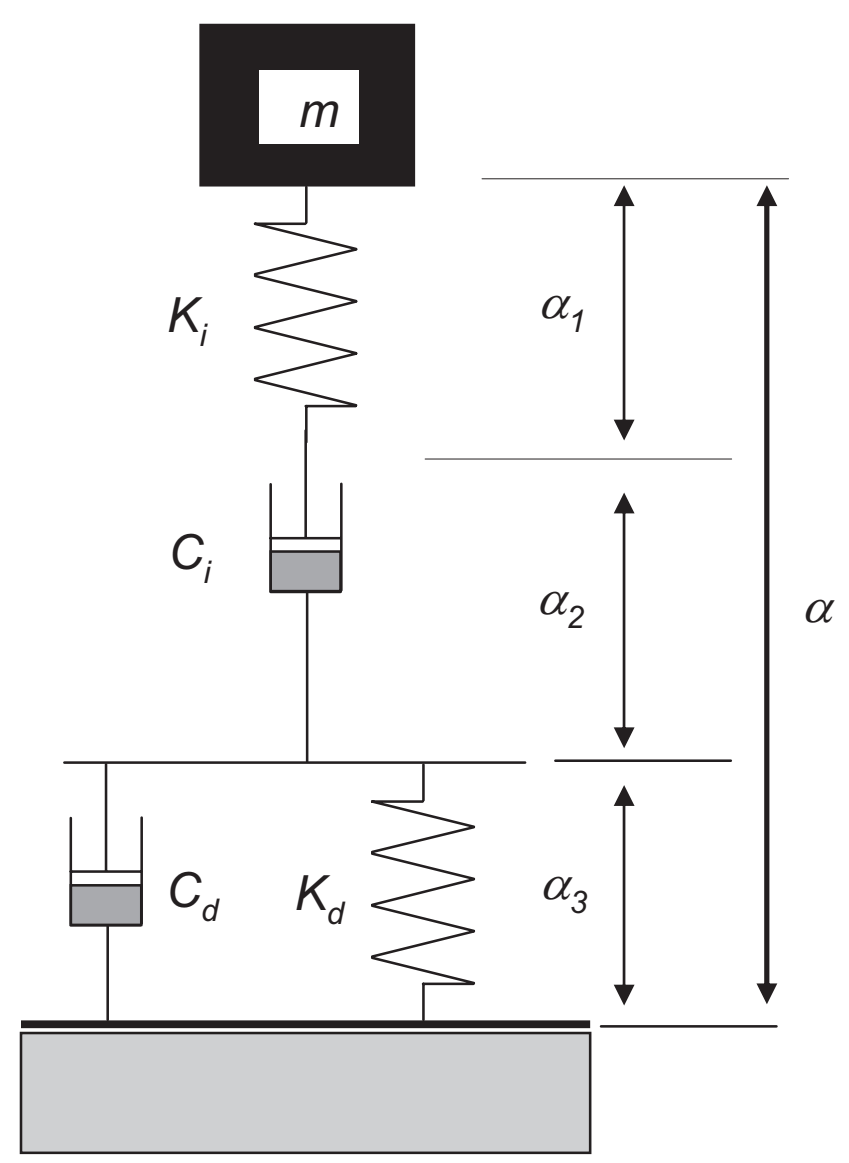

FIGURE 3.

Scheme of the lumped mass spring model.

$$
\begin{gathered}
\mathrm{F}_{\mathrm{K}_{\mathrm{d}}}=\mathrm{K}_{\mathrm{d}} \alpha_{3} \\
\mathrm{~F}_{\mathrm{C}_{\mathrm{d}}}=\mathrm{C}_{\mathrm{d}} \dot{\alpha}_{3} \\
\mathrm{~F}(\mathrm{t})=-\mathrm{F}_{\mathrm{K}_{\mathrm{i}}}=-\mathrm{F}_{\mathrm{C}_{\mathrm{i}}}=-\mathrm{F}_{\mathrm{K}_{\mathrm{d}}}-\mathrm{F}_{\mathrm{C}_{\mathrm{d}}}
\end{gathered}
$$

where symbol $\alpha$ is the total displacement, and $\alpha_{1}, \alpha_{2}$, and $\alpha_{3}$ are the partial displacements of the model elements, as shown in Fig. 3. $\dot{\alpha}$ corresponds to the dart velocity.

Previous work has shown the best experimental fitting was obtained when the two mechanisms are placed in a serial disposition, whereas the spring and the damper in the flexural mechanism work in parallel. ${ }^{8}$

For isotropic elastic materials, the spring constant $K_{d}$ is a function of the test geometry. In the case of a circular simple supported plate centrally loaded, the vending stiffness is given by?

$$
\mathrm{K}_{\mathrm{d}}=\frac{\mathrm{F}}{\alpha}=\frac{4 \pi}{3} \cdot \frac{\mathrm{d}^{3}}{(1-v)(3+v) \mathrm{R}^{2}} \cdot \mathrm{E}
$$

where $\mathrm{d}$ is the specimen thickness, $\mathrm{R}$ the support radius, $v$ the Poisson coefficient, and $\mathrm{E}$ the elastic modulus.

The load is a linear function of the plate deflection and the tensile stress at the opposed face of the impact can be obtained from the applied stress as ${ }^{10}$ :

$$
\sigma=\frac{\mathrm{F}}{\mathrm{d}^{2}}\left[(1+v) \cdot\left(0.485 \cdot \log \left(\frac{\mathrm{R}}{\mathrm{d}}\right)+0.52\right)+0.48\right]
$$

When the specimen breaks, then the tensile stress at the failure load can be readily found.

Similarly, $\mathrm{K}_{\mathrm{i}}$ can be related to elastic modulus $\mathrm{E}$ using Hertz law:

$$
\mathrm{K}_{\mathrm{i}}=\frac{4}{3} \cdot \frac{\sqrt{\mathrm{r}}}{\left(1-v^{2}\right)} \cdot \mathrm{E}
$$

where $r$ is the indenter radius.

\section{RESULTS AND DISCUSSION}

A series of impact tests were performed on each material at increasing impact energies, i.e., increasing drop heights (h). Each specimen was tested only once. Although a continuous curve was registered for the lower energy impacts, as it can be seen in Fig. 4, failure was reached for the higher energy impacts and the curves showed a sudden decrease of load values, as shown in Fig. 5. Different types of information can be subtracted from each of these two groups of specimens. The former can give information about material response under non-failure conditions, such as stiffness and energy dissipation. The latter can give information about material failure limit and energy it can absorb before this limit is reached.

\section{Non-Failure Conditions}

By using Eq. 7 on the raw data of the specimens that did not fail, it was possible to obtain the experimental restitution coefficient. All the impacts for both materials showed values smaller than 1 , 


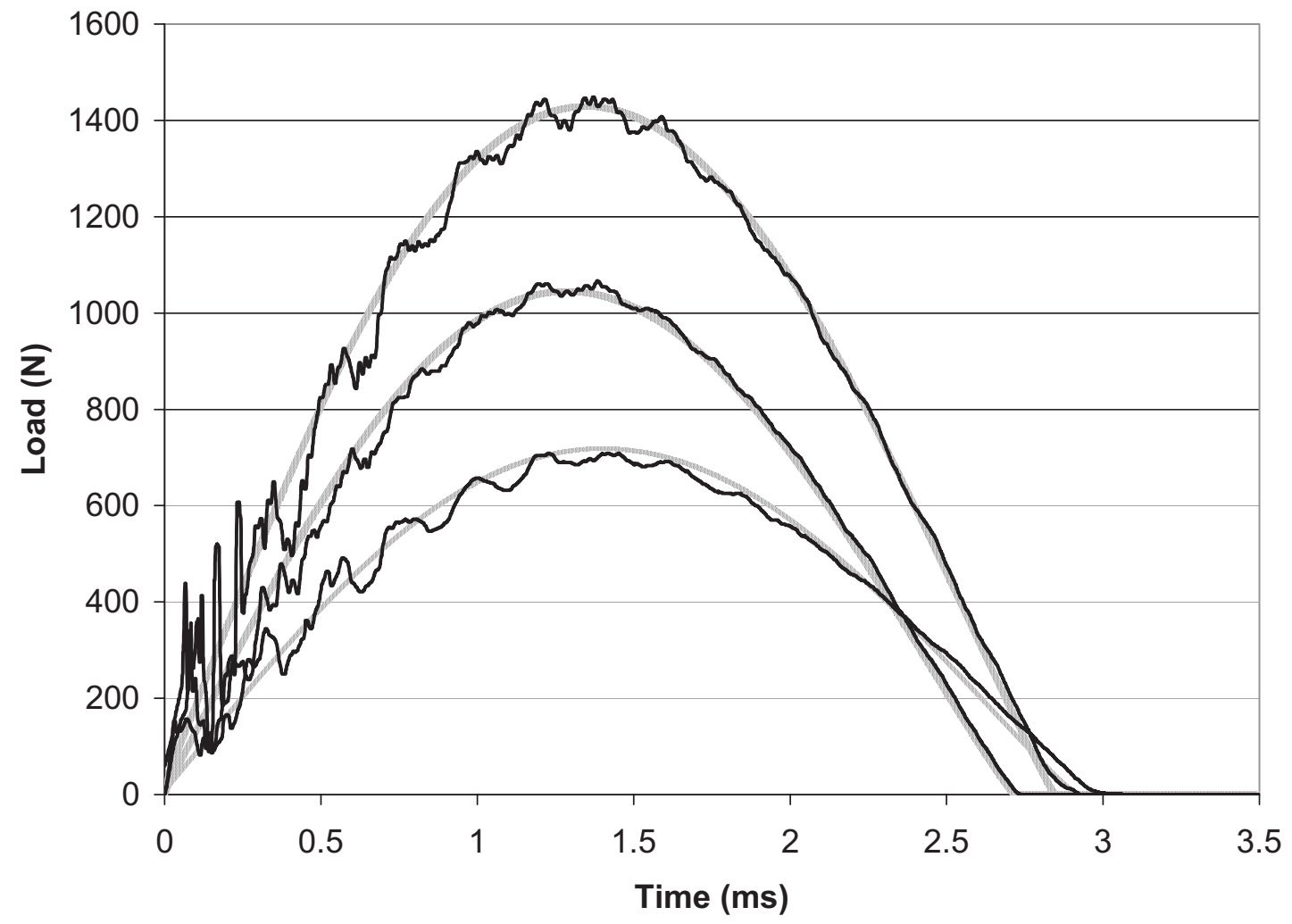

FIGURE 4.

Load vs. time for CR-39 specimens that did not fail, impacted at three different velocities. Gray lines show model prediction.

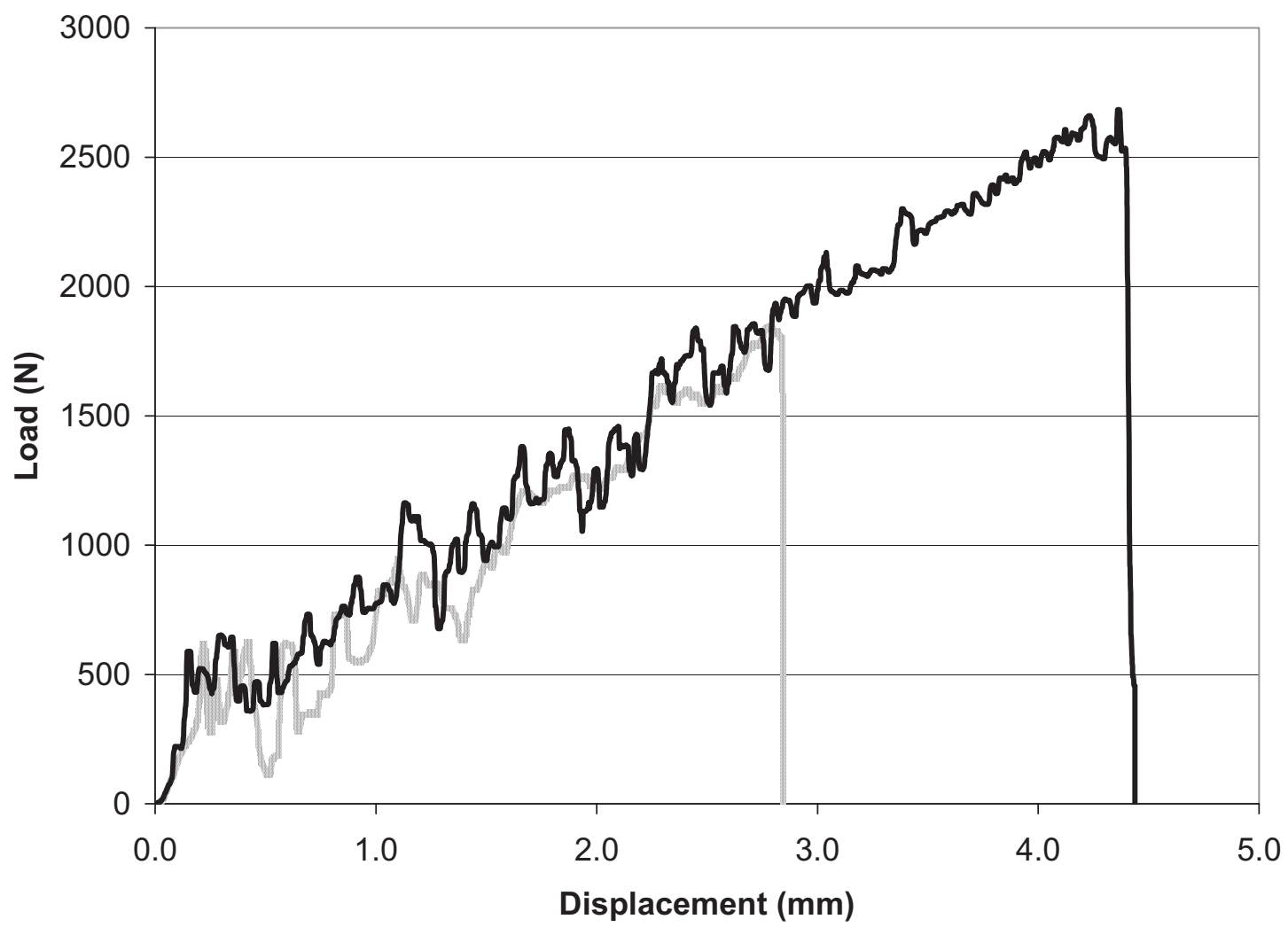

FIGURE 5.

Examples of load vs. displacement for specimens that failed of Superfin (black line) and CR-39 (gray line). 


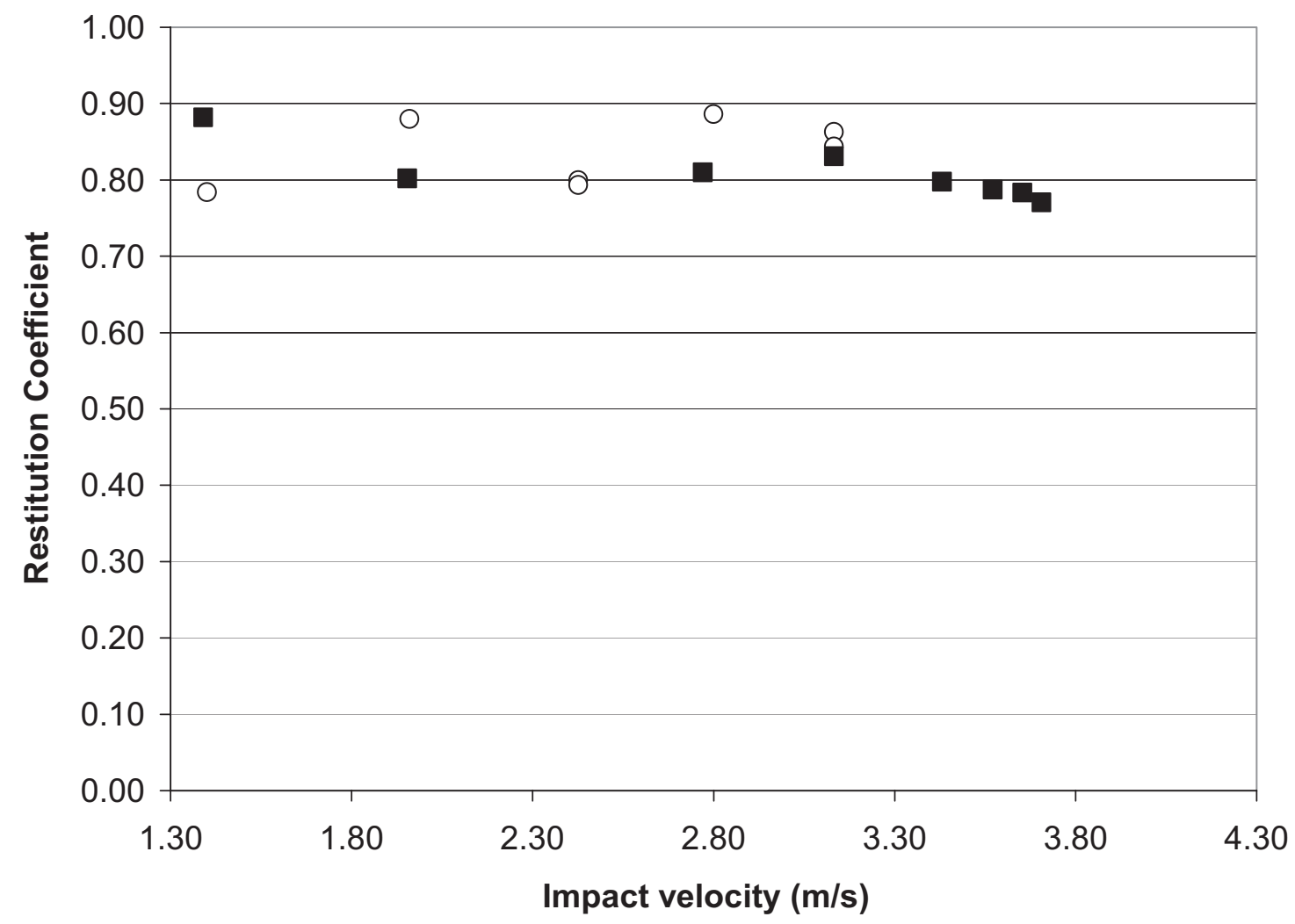

FIGURE 6.

Restitution coefficient in front of initial velocity for Superfin (white) and CR-39 (black).

\section{TABLE 1.}

Experimental and numerical results of the low energy impacts for CR-39 and Superfin $\left(\mathrm{F}_{\mathrm{m}}=\right.$ maximum load; $\alpha_{\mathrm{m}}=$ maximum indentation; $\varepsilon=$ restitution coefficient; $\mathrm{E}=$ elastic modulus; $\mathrm{C}_{\mathrm{i}}=$ indentation damper coefficient; $\mathrm{C}_{\mathrm{d}}=$ deflection damper coefficient)

\begin{tabular}{|c|c|c|c|c|c|c|c|c|c|c|}
\hline & \multirow[b]{2}{*}{$\nu_{\mathrm{O}}(\mathrm{m} / \mathrm{s})$} & \multicolumn{3}{|c|}{ Experimental } & \multicolumn{6}{|c|}{ Numerical } \\
\hline & & $F_{m}(\mathrm{~N})$ & $\alpha_{m}(\mathrm{~mm})$ & $\varepsilon$ & $\mathrm{F}_{m}^{\prime}(\mathrm{N})$ & $\alpha_{m}^{\prime}(\mathrm{mm})$ & $\varepsilon^{\prime}$ & $E(\mathrm{GPa})$ & $C_{i}(\mathrm{Kg} / \mathrm{s})$ & $C_{d}(\mathrm{Kg} / \mathrm{s})$ \\
\hline \multirow[t]{3}{*}{ CR-39 } & 1.39 & 709 & 1.28 & 0.88 & 700 & 1.28 & 0.90 & 3.30 & 8,000 & 10 \\
\hline & 1.95 & 1067 & 1.77 & 0.80 & 1045 & 1.77 & 0.80 & 3.40 & 4,500 & 10 \\
\hline & 2.80 & 1448 & 2.59 & 0.81 & 1412 & 2.61 & 0.81 & 3.42 & 5,000 & 15 \\
\hline \multirow[t]{3}{*}{ Superfin } & 1.39 & 743 & 1.31 & 0.92 & 702 & 1.36 & 0.92 & 3.27 & 11,000 & 10 \\
\hline & 1.96 & 1009 & 1.76 & 0.88 & 987 & 1.82 & 0.88 & 3.35 & 8,500 & 15 \\
\hline & 2.80 & 1530 & 2.56 & 0.86 & 1429 & 2.63 & 0.86 & 3.40 & 7,000 & 15 \\
\hline
\end{tabular}

indicating energy losses and that a better representation of the system dynamics would be with non-conservative models.

A plot of this parameter in front of the impact velocity (Fig. 6) showed that as the velocity increased the restitution coefficient decreased, as it can be expected for polymers materials with a viscoelastic nature.

Adjusting the elastic modulus $E$ and damper coefficients $\mathrm{C}_{\mathrm{d}}$ and $\mathrm{C}_{\mathrm{i}}$ in the full model, it was possible to generate a numerical curve of load in front of the time. As it can be observed in Fig. 4, the numerical curve satisfactorily fitted the experimental data. High frequency oscillations on the experimental curve came from dart head and sample plate vibrations, which have not been included in the model.

Table 1 shows the most significant fitting parameters for the lower energy impacts, which are the ones that satisfy the small deflection criteria. Maximum loads $\left(\mathrm{F}_{\mathrm{m}}\right)$ were directly read from the raw data. As expected, registered and calculated maximum load increased as the impact energy increased and the registered differences fall within the experimental error.

Using Eq. 2, the displacement of the dart could be calculated for experimental and numerical curves. As it was also expected, larger energy impacts resulted in larger maximum dart displacement values. A very good agreement was also found between calculated and experimental data.

In a similar manner as was performed on experimental data, numerical restitution coefficients could be calculated using Eq. 7 . Data in Table 1 shows numerical and experimental values are also in good agreement. Unsurprisingly, larger impact energies gave larger maximum contact forces and larger deflections, which 
caused an increase in the energy dissipation. As expected, the nonconservative model also shows good concordance with this behavior.

After the testing, an imprint on the specimen surface could be observed, which was originated by the contact of the striker head with the specimen. This imprint is a consequence of the plastic and viscoelastic deformation in the contact and increased with striker available energy.

After optimal fitting was achieved, elastic modulus for both materials could be compared. Results are also shown in Table 1. Both materials showed similar elastic modulus values. The slight increase in this parameter as the velocity increased for each material can be attributed to the viscoelastic nature of polymers.

The values of damper coefficients $\mathrm{C}_{\mathrm{i}}$ and $\mathrm{C}_{\mathrm{d}}$ adjusted during the fitting process are also shown in Table 1 . The difference in the damper values of the indentation and deflection mechanisms is quite remarkable since indentation damper coefficients showed values from $4500 \mathrm{~kg} / \mathrm{s}$ to $11,000 \mathrm{~kg} / \mathrm{s}$, whereas deflection damper coefficients stayed in the range of 10 to $15 \mathrm{~kg} / \mathrm{s}$.

An analysis of the damper coefficients can give a further understanding of the nature of the mechanism that causes the energy dissipation. In non-conservative systems, restitution coefficient is always smaller than 1 , and energy dissipation is modeled through the dampers. However, when a dashpot works in serial disposition, as in the case of the indentation mechanism, the smaller its coefficient value, the larger the energy dissipated. Contrarily, if a damper works in parallel to a spring, as in the case of the plate deflection mechanism, a small coefficient values imply smaller energy dissipation.

Therefore, in this work, damper coefficients reveal that energy dissipation mainly takes place by means of the indentation mechanism because $\mathrm{C}_{\mathrm{d}}$ values are almost negligible. Indeed, deflection coefficients are probably 0 but they could not be introduced as so into the model because of limitations of the mathematical method. As expected for small deflection plate theory, the deflection mechanism is conservative in this range and only the indentation damper is actuating, causing the non-conservative behavior of the material. Moreover, as the impact velocity increases, the dissipated energy becomes larger, which becomes evident in a decreasingly smaller value of the restitution coefficient and a smaller value of the indentation damper coefficient $\mathrm{C}_{\mathrm{i}}$, as well.

\section{Failure Conditions}

Another interesting aspect that can give a more through understanding of material behavior and that can be used to compare materials is the analysis of their failure limit in terms of rupture stress and absorbed energy at failure.

Contact time $\left(t_{c}\right)$ is a measurement of the time the dart tip remains in contact with the specimen during the impact. The plot of this parameter vs. initial dart velocity for each specimen allowed distinguishing the ones that remained intact, which showed larger contact time values, from the ones that failed, with smaller values. Because this transition is sharp enough, it is possible identify at what initial velocity the material started to fail and the rupture load could be obtained from the plot of the maximum load $\mathrm{F}_{\mathrm{m}}$ in front of the initial $\mathrm{v}_{0}$ velocity (Figs. 7 and 8 ).

Because of different material response, drop weights used for CR-39 and Superfin were different. However, failure limit could

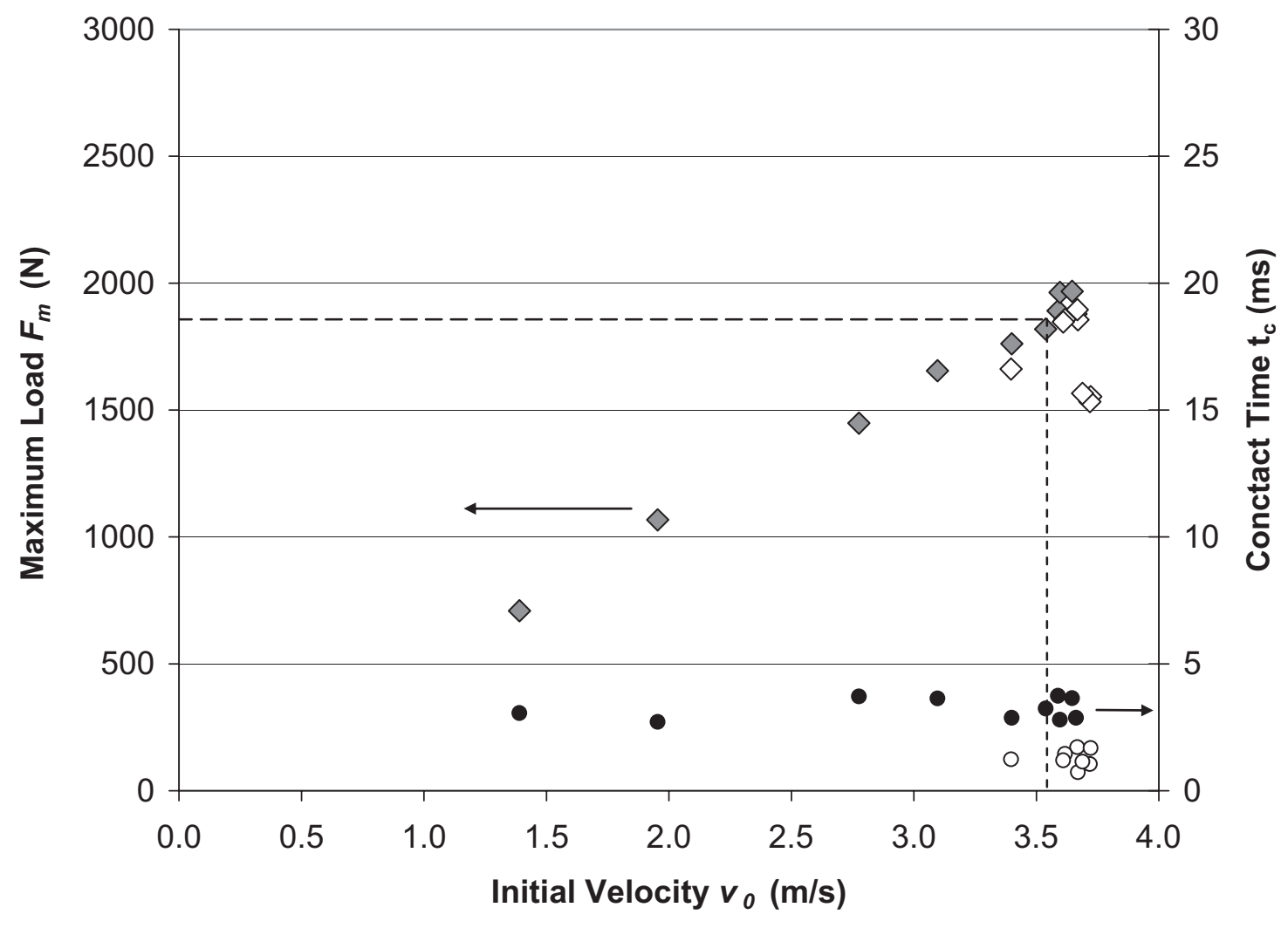

FIGURE 7.

Maximum load $\mathrm{F}_{\mathrm{m}}(\boldsymbol{)})$ and contact time $\mathrm{t}_{\mathrm{c}}$ specimens that failed. Dashed line shows initial velocity at contact time decrease and its corresponding rupture load. 


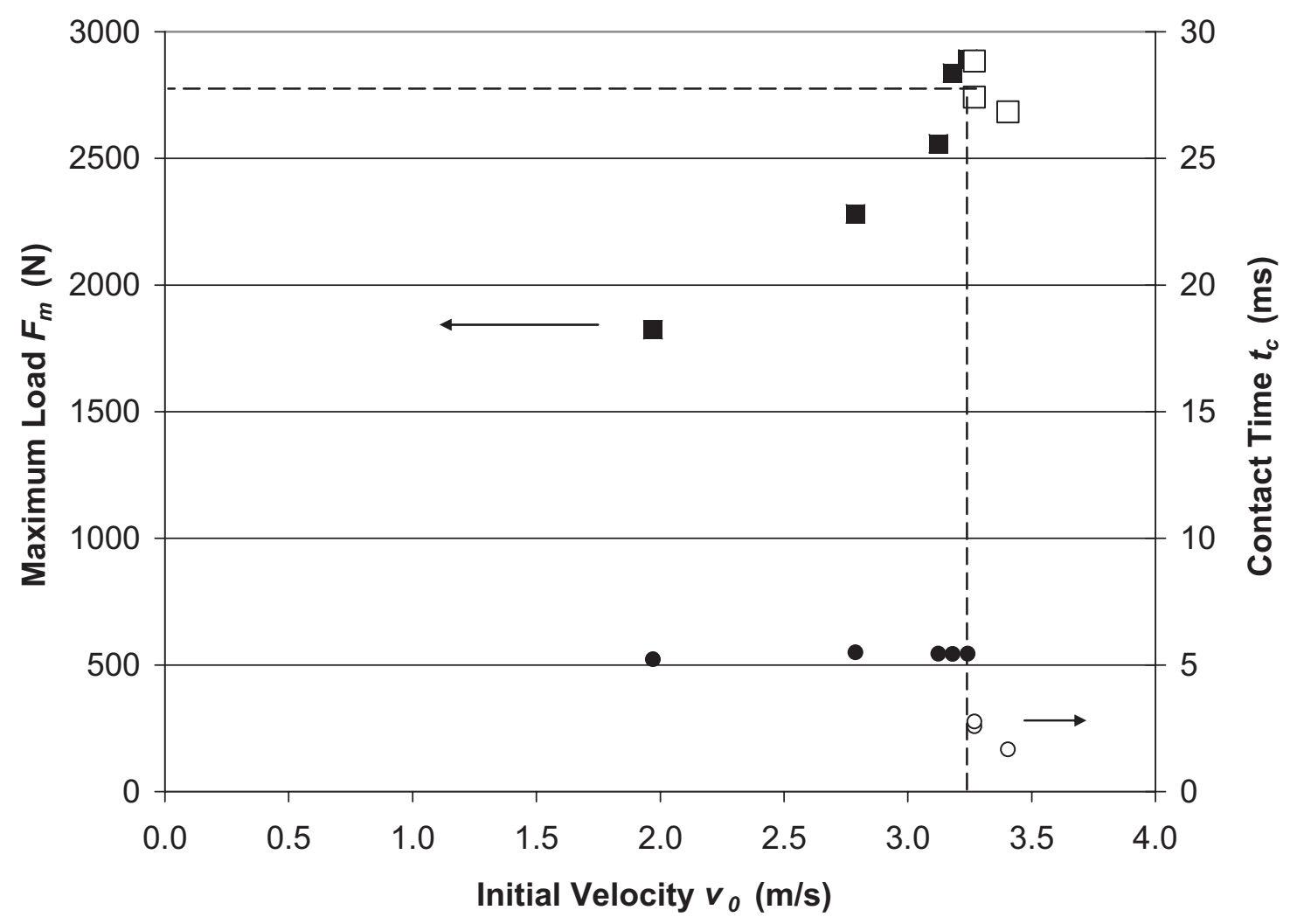

\section{FIGURE 8.}

Maximum load $F_{m}$

and contact time $t_{c}$

vs. striker initial velocity $v_{0}$ for Superfin. Striker load was $1.504 \mathrm{~kg}$. Black, specimens that did not fail; white, specimens that failed. Dashed line shows initial velocity at contact time decrease and its corresponding rupture load.

TABLE 2.

Rupture stress $\left(\sigma_{\mathrm{r}}\right)$, absorbed energy calculated using initial velocity $\left(\mathrm{E}_{\mathrm{a}}\right)$ and absorbed energy calculated by integration $\left(E_{\mathrm{a}}^{\prime}\right)$ of CR-39 and Superfin

\begin{tabular}{lccc}
\hline & $\sigma_{r}\left(\mathrm{~N} / \mathrm{mm}^{2}\right)$ & $\mathrm{E}_{\mathrm{a}}(J)$ & $E_{\mathrm{a}}^{\prime}(J)$ \\
\hline CR-39 & 140 & 3.1 & 2.7 \\
Superfin & 216 & 7.9 & 7.5 \\
\hline
\end{tabular}

be readily achieved for CR-39 using a $0.503 \mathrm{~kg}$ drop weight and progressively increasing the falling height for each tested specimen, a $1.504 \mathrm{~kg}$ drop weight was needed for Superfin to reach the failure limit within the equipment falling height range.

Using this method, a rupture load of $1800 \mathrm{~N}$ at an initial velocity of $3.6 \mathrm{~m} / \mathrm{s}$ was obtained for CR-39, and a value of $2750 \mathrm{~N}$ for Superfin at an initial velocity of $3.25 \mathrm{~m} / \mathrm{s}$ was obtained.

The plot of the registered load in front of the dart displacement can reveal the type of dependence of these two parameters. If this relationship is linear, Eq. 14 can be applied and the obtained slope would be equal to the deflection spring constant $\mathrm{K}_{\mathrm{d}}$. As a consequence, Eq. 15 can be used to calculate the stress at the opposite face of the plate. When rupture load was used in this equation, then the rupture stress $\sigma_{\mathrm{r}}$ was obtained.

Plot of the registered load in front of the dart displacement showed very linear dependence for both Superfin and CR-39. Examples are plotted in Fig. 5. When the rupture load that had been previously obtained was used (1800 N for CR-39 and $2750 \mathrm{~N}$ for Superfin), then the rupture stress $\sigma_{\mathrm{r}}$ could be obtained for each material. Results are shown in Table 2. Rupture stress for Superfin gave a value $54 \%$ larger than the one of CR39.

Regarding the absorbed energy at the failure limit, two methods can be used to calculate it. The first one involves using the initial velocity values at maximum failure load $\mathrm{F}_{\mathrm{m}}$ and carrying out a simple calculation of the absorbed kinetic energy $\mathrm{E}_{\mathrm{a}}$ :

$$
\mathrm{E}_{\mathrm{a}}=\frac{1}{2} \mathrm{~m} v_{0 \text { fail }}^{2}
$$

Using Eq. 17, absorbed kinetic energy was calculated for CR-39 with a $\mathrm{v}_{0 \text { fail }}=3.6 \mathrm{~m} / \mathrm{s}$ and a mass of $0.503 \mathrm{~kg}$, and in the case of Superfin a $\mathrm{v}_{0}$ fail $=3.25 \mathrm{~m} / \mathrm{s}$ and a mass of $1.504 \mathrm{~kg}$. As it is displayed in Table 2, Superfin obtained a value $170 \%$ lager than CR-39.

These results could be contrasted by using a second calculation method of the absorbed energy $E_{a}^{\prime}$. It involves integrating the area below the maximum load vs. displacement plot for all the failed specimens (Fig. 5) to obtain an averaged value of the absorbed energy.

Values calculated through this method are also shown in Table 2. In this case, the energy increase of Superfin over CR-39 was of $178 \%$. Agreement among both methods is quite remarkable.

As can be seen in this article, instrumented equipment is a valid tool for obtaining the absorbed impact energy and studying when the damage initiates in a specimen. This methodology can also be applied to specimens with any given shape and, of course, real ophthalmic lenses could also be used. However, the presented model and the tensile stress at failure expressions are only valid if 
plate-shaped specimens are used because corresponding equations where calculated assuming this particular geometry. Other geometries would make mathematical expressions very difficult to handle, if solvable at all, and Runge-Kutta methods would not be sufficient.

In addition, this methodology of low energy impacts on plateshaped specimens has been shown to be very useful to simultaneously determine the point when damage initiates, the tensile stress at failure, the elastic modulus, and the failure energy under impact conditions very similar to those a real lens can suffer, which makes this procedure specially suitable to compare and optimize ophthalmic materials.

\section{CONCLUSIONS}

Low energy impact methods are a very powerful tool to compare ophthalmic materials. A model for predicting the contact duration, force, indentation, and displacement under impact has been developed for impact a plate with a hemispherical indenter. The governing equations were first proposed and then solved.

Comparisons between the model predictions and the experimental results are in very good agreement. Comparison of the impact force history also shows good accord. Both tested materials showed non-conservative impact response and could be successfully modeled using a lumped mass spring model. Calculated numerical curves were in good agreement with registered experimental curves and values.

Using this method, elastic modulus and damper coefficients could be readily obtained for Superfin and CR-39 materials. Although these two materials showed similar values in their elastic modulus, slightly lower damper coefficients were obtained for CR39 , which is indicative of a larger amount of energy dissipation during impact.

In the failure analysis, a 54\% larger rupture stress value was calculated for Superfin, which makes this material significantly more impact resistant than CR-39. Moreover, absorbed energy to failure clearly favored Superfin over CR-39. Indo's proprietary material showed energy values more than two and a half times larger than those of CR-39 in both calculation methods.

The monomer composition of Superfin, with a $50 \%$ of aromatic polyester oligomer, could explain the observed differences because the presence of an oligomer implies lower cross-linking density, which allows internal reorientation of the polymer chains during impact. This could lead to a larger deformation before crack formation starts, giving greater maximum load values and allowing a larger amount of absorbed energy before failure is reached.

Received December 16, 2008; accepted March 9, 2009.

\section{REFERENCES}

1. International Organization for Standardization. Plastics-Determination of Puncture Impact Behaviour of Rigid Plastics-Part 2: Instru- mented Puncture Test. ISO 6603-2; 2000. Geneva: International Organization for Standardization; 2000.

2. Ward IM, Hadley DW. Mechanical Properties of Solid Polymers, 2nd ed. New York: Wiley; 1933.

3. Williams JG. Fracture Mechanics of Polymers. Chichester: E. Horwood; 1984.

4. Kinloch AJ, Young RJ. Fracture Behaviour of Polymers. Dordrecht: Kluwer Academic Publishers; 1983.

5. U.S. Department of Health and Human Services, Food and Drug Administration. Food and Drug Administration (FDA) Test Standard 21 CFR 801.410(d)(2). Washington, DC: U.S. Department of Health and Human Services, Food and Drug Administration; 2000.

6. Chou BR, Gupta A, Hovis JK. The effect of multiple antireflective coatings and center thickness on resistance of polycarbonate spectacle lenses to penetration by pointed missiles. Optom Vis Sci 2005;82: 964-9.

7. Jiménez O, Sullcahuaman JA, Sánchez-Soto M, Martínez AB. Low energy impact evaluation using non conservative models. Int J Sol Struct 2005;42:5758-64.

8. Artús P, Dürsteler JC, Martínez AB. Low-energy dynamic indentation method for analysis of ophthalmic materials. Optom Vis Sci 2008;85:49-53.

9. Timoshenko S, Goodier JN. Theory of Elasticity, 3rd ed. New York: McGraw-Hill; 1982.

10. Timoshenko SP, Woinowsky-Krieger S. Theory of Plates and Shells, 2nd ed. New York: Mc Graw-Hill; 1969.

11. Nobre JP, Dias AM, Gras R. Resistance of a ductile steel surface to spherical normal impact indentation: use of a pendulum machine. Wear 1997;211:226-36.

12. Southern E, Thomas AG. The impact of rigid spheres on rubber. J Appl Polym Sci 1972;16:1641-51.

13. Zukas JA, Greszczuk JB. Recent Advances in Impact Dynamics of Engineering Structures. Malabar, FL: Krieger Pub. Co.; 1992.

14. Vriend NM, Kren AP. Determination of the viscoelastic properties of elastomeric materials by the dynamic indentation method. Polym Test 2004;23:369-5.

15. Rydin RW, Karbhari VM. Partitioning energy during low-velocity impact of rtm fiber-reinforced composites. Int J Impact Eng 1998; 21:773-89.

16. Martínez AB, Sánchez-Soto M, Velasco JI, Maspoch ML, Santana OO, Gordillo A. Impact characterization of carbon fiber-epoxy laminate using a nonconservative model. J Appl Polym Sci 2005;97: 2256-63.

17. Sánchez-Soto M, Martínez AB, Santana OO, Gordillo A. On the application of damped model to the falling weight impact characterization of glass beads-polystyrene composites. J Appl Polym Sci 2004; 93:1271-84.

18. Martinez AB, Agulló J, Jiménez O, Sullcahuaman JA, Sánchez-Soto M, Maspoch ML, Velasco JI, Santana OO, Gordillo A. Impacto de baja energía en composites de poliestireno. Gijón, Spain: Congreso Nacional de Materiales Compuestos; 2001.

Pau Artús

Indo Lens Group S.L. C/Alcalde Barnils 72 08174 Sant Cugat del Vallès Spain e-mail:pau.artus@indo.es 